\title{
Autoevaluación sobre formación en medicina paliativa en una cohorte de residentes
}

\author{
Paula Vial $C^{1 a}$, Patricio Ibáñez $\mathrm{L}^{1 \mathrm{a}}$, Alfredo U maña $\mathrm{V}^{2 b}$, \\ María Margarita Reyes D ${ }^{3 C}$, Paola Viviani ${ }^{3 d}$, Flavio Nervi 0 1,4,5. \\ Self assessment about proficiency on \\ palliative care in a cohort of \\ residents
}

Background: Little is known about the status and level of medical education on palliative care, symptom control and aspects of end of life care provided by medical school programs in Chile. Aim: To determine if a cohort of residents had palliative care and end of life care education during pre graduate and post graduate training. Residents were also asked to self assess their proficiency in these areas. Material and Methods: In 2002, we contacted 200 of a total of 327 residents of the postgraduate programs of the Pontificia Universidad Catolica de Chile. They were asked to anonymously answer a preformed questionnaire addressing different aspects related to palliative care, symptom control and end of life issues. Results: Less than $25 \%$ of residents received some degree of palliative care education during their studies, and approximately half of them considered that the level of proficiency reached was less than $25 \%$. This contrasted with a good self assessment in the management of pain and gastrointestinal symptoms. For the treatment of the anorexia-cachexia syndrome and delirium, approximately $50 \%$ of the cohort had a mean self assessment score of 3 in a scale of 1 to 7 . More than $80 \%$ of residents considered that palliative medicine education is important for their medical career. Conclusions: Chilean medical schools must improve palliative care and end of life training as part of their mandatory curriculum in both the pre and post graduate levels (Rev Méd Chile 2004; 132: 445-52).

(Key Words: Education, medical, graduate; Palliative care; Residency, medical; Terminally ill)

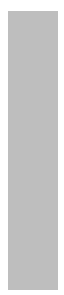

Recibido el 5 de agosto, 2003. Aceptado en versión corregida el 6 de enero, 2004.

Trabajo financiado por Proyecto ICU/Chile 1091-G224 y Fundación Pro Dignitate Hominis. Departamentos de Gastroenterología ${ }^{1}$ y de Medicina ${ }^{2}$, y de Salud Pública ${ }^{3}$, Facultad de Medicina y Centro de Bioética ${ }^{4}$, Pontificia Universidad Católica de Chile y Clínica Familia para Cuidados Paliativos ${ }^{5}$.

aBecaria de Gastroenterología, bBecario de Medicina Interna; 'Psicóloga y Enfermera Uni-

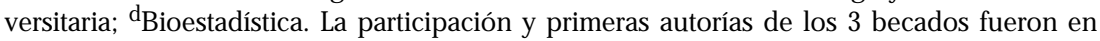
todo equivalentes.

Correspondencia a: Dr. Flavio Nervi. Pontificia Universidad

Católica de Chile, Departamento de Gastroenterología.

Marcoleta 367, Santiago. Teléfono 6863822. Fax 6397780.

E-mail: fnervi@med.puc.cl 
$\mathrm{E}^{1}$ curnículo de las escuelas de medicina ha incluido los cuidados paliativos* en los capítulos de psiquiatría, manejo del dolor y otros síntomas sistémicos y variados cursos de formación general a lo largo de los estudios de pregrado ${ }^{1-4}$. El énfasis puesto en estas áreas se ha fundamentado en la observación de que el paradigma científico-tecnológico de la medicina exitosa de los tiempos modernos, no es suficiente para satisfacer las demandas de la población, especialmente en relación con el sufrimiento humano y la muerte ${ }^{5-11}$. Con el objeto de reforzar la formación médica en los aspectos humanísticos para satisfacer las demandas de la sociedad, las escuelas de medicina han implementado cambios en sus curículos. Se han introducido así cursos de humanización de la medicina, bioética y antropología entre otros, en el contexto de la atención médica de pacientes con enfermedades crónicas incurables y cercanos a su muerte, tanto en Chile $^{12-20}$ como en el extranjero ${ }^{21-27}$.

El control de síntomas de los pacientes incurables, incluidos los pacientes con cáncer metastásico, SIDA, accidentes vasculares cerebrales, demencia y aquellos portadores de insuficiencias hepáticas, cardíacas, respiratorias o renales descompensadas, se ha desarrollado en 3 instancias diferentes, pero no excluyentes ${ }^{3,23}$. Primero, a nivel de la atención primaria por médicos generales. En ocasiones, esta labor se da en el contexto de equipos multidisciplinarios que incluyen además de médicos, enfermeras, psicólogos, sacerdotes, rabinos o pastores, y voluntarios entrenados. Una segunda instancia incluye la participación de especialistas en oncología, anestesiología, cirugía y radioterapia, los cuales ven a los enfermos desde la perspectiva del control de síntomas, especialmente participando en la planificación de terapias orientadas al control del dolor.

Un tercer nivel de atención incluye a médicos que han recibido un entrenamiento específico en medicina paliativa* ${ }^{* 28}$, orientado desde una filosofía holística, incluyendo todas las áreas del sufrimiento humano (〈dolor total»), con los componentes físico, psicológico, social y espiritual ${ }^{1,3,5-7}$.
La forma de abordar esta área emergente de la medicina contemporánea es variable en distintos países $^{24}$. La medicina y cuidados paliativos se han desarmollado a partir de la década 1960-69, en Inglaterra ${ }^{1,3}$ y posteriormente en otros países desarmollados $^{3}$. Esta es una especialidad reconocida en Inglaterra desde $1987^{27}$ y su enseñanza es obligatoria en las escuelas de medicina de ese país ${ }^{23}$, no así en otros países desamollados ${ }^{25}$. La situación en Chile, sobre educación en medicina y cuidados paliativos es poco conocida ${ }^{29,30}$. Ello tiene importancia si se considera un aumento considerable de la población mayor de 65 años en los próximos años ${ }^{31,32}$.

El objetivo de este trabajo fue, primero, determinar qué proporción de una cohorte de médicos residentes, que están desarrollando sus programas de postítulo, tuvo formación específica en cuidados paliativos y control de síntomas, durante los estudios de pre y post grado. Segundo, conocer su impresión subjetiva sobre el nivel de capacitación alcanzado en relación con la atención de pacientes incurables que están por morir.

\section{MATERIAL Y MÉTODO}

Del total de 327 residentes de especialidades primarias (medicina, cirugía, obstetricia, pediatría, medicina y pediatría ambulatoria, anatomía patológica, neurología, traumatología, radiología y laboratorio clínico) y subespecialidades matriculados en la escuela de postgrado de la Facultad de Medicina de la Pontificia Universidad Católica de Chile durante 2002, se contactaron 200 que se encontraban haciendo sus rotaciones por el Hospital Clínico. Aproximadamente un tercio de los residentes hicieron sus estudios de pregrado en la Universidad de Chile, 40\% en la Pontificia Universidad Católica de Chile, 20\% en universidades de provincias y el resto en otras universidades incluyendo extranjeras y privadas nacionales. Debido al número relativamente pequeño de individuos provenientes de numerosas escuelas de medicina, sólo se hicieron comparaciones entre los residen-

* La Organización Mundial de la Salud define los Cuidados Paliativos como el cuidado activo y total de los pacientes portadores de enfermedades que no responden a tratamiento curativo. El control del dolor y de otros síntomas, así como la atención de aspectos psicológicos, sociales y espirituales es primordial. Ofrece un sistema de apoyo para ayudar a los pacientes a vivir tan activamente como sea posible hasta la muerte. Apoya a la familia en la enfermedad del paciente y durante el duelo ${ }^{2}$.

* Medicina paliativa es el estudio y tratamiento de pacientes portadores de enfermedades activas, progresivas y avanzadas, para los cuales el pronóstico es muy limitado y el énfasis terapéutico está puesto en la calidad de vida ${ }^{23}$. 
tes que hicieron sus estudios de pregrado en la Universidades de Chile, Pontificia Universidad Católica y las de provincia como un solo grupo.

Con el objeto de comparar las áreas médicas de donde provenían, los residentes fueron agrupados arbitrariamente en 3 áreas: a) médica (medicina interna y subespecialidades, pediatría y medicina ambulatoria); b) quirúrgica (cirugía y subespecialidades, traumatología y obstetricia-ginecología); c) diagnóstica (radiología, patología y laboratorio clínico).

Cuestionario. Se elaboró un cuestionario de 25 preguntas sobre aspectos cualitativos y cuantitativos de la capacitación alcanzada en cuidados paliativos, incluyendo: 1. Exposición a cursos y seminarios de cuidados paliativos, y autocalificación del nivel de formación alcanzado. 2. Formación sobre aspectos antropológicos y éticos relacionados con la muerte y calificación de la capacidad de enfrentarse a los problemas del paciente terminal y su familia. 3. Grado de capacitación alcanzado en control de síntomas de enfermos portadores de enfermedades incurables en fase avanzada. Los síntomas analizados fueron agrupados en 3 grupos sistémicos, incluyendo: dolor, astenia-caquexia y anorexia; síntomas gastrointestinales incluidos la náusea, constipación y diarrea, y los síntomas psiquiátricos más comunes como angustia, depresión y delirio. 4. Opinión sobre la importancia de los cuidados paliativos para su formación médica y práctica profesional.

Las encuestas fueron entregadas individualmente por el Jefe de Beca correspondiente a cada uno de los programas. Estas fueron contestadas de manera anónima y la información fue digitada en un sistema computacional para el análisis estadístico de las respuestas.

Estadística. Las diferencias entre los grupos se evaluaron utilizando el test de t de Student o el test de Chi cuadrado con corrección de Fisher donde correspondía.

\section{RESULTADOS}

El $64 \%$ de los 200 residentes correspondió a hombres y el $36 \%$ a mujeres. Un tercio estaban haciendo el primer año de beca, un tercio el segundo o tercero de una beca de especialidad primaria y un tercio, una beca de subespecialidad. El 44\% habían egresado de la escuela de medicina antes del año 1997, un tercio entre el 98 y 99, y $25 \%$ el 2000. Un tercio tenían al menos tres años de ejercicio profesional (Tabla 1).

Tabla 1. C aracterísticas de la población de residentes estudiados

\begin{tabular}{|c|c|c|c|c|c|c|c|}
\hline \multirow[t]{2}{*}{ Característica } & & \multicolumn{2}{|c|}{ Mujeres } & \multicolumn{2}{|c|}{ Hombres } & \multicolumn{2}{|c|}{ Total } \\
\hline & & $\mathrm{n}$ & $(\%)$ & $\mathrm{n}$ & $(\%)$ & $\mathrm{n}$ & (\%) \\
\hline \multirow[t]{4}{*}{ Tipo de residencia* } & Médica & 55 & (50) & 56 & (50) & 111 & (55) \\
\hline & Quirúrgica & 9 & (13) & 60 & (87) & 69 & (35) \\
\hline & Diagnóstica & 7 & (35) & 13 & (65) & 20 & (10) \\
\hline & Total & 71 & (36) & 129 & (64) & 200 & (100) \\
\hline \multirow[t]{4}{*}{ Año de residencia** } & Primero & 19 & (30) & 44 & (70) & 63 & (44) \\
\hline & Segundo & 20 & (45) & 24 & (55) & 44 & (31) \\
\hline & Tercero & 14 & (39) & 23 & (61) & 37 & (25) \\
\hline & Total & 53 & (37) & 91 & (63) & 144 & (100) \\
\hline \multirow[t]{4}{*}{ Año de egreso } & $\leq 1997$ & 25 & (29) & 60 & (71) & 85 & (44) \\
\hline & 1998-99 & 26 & (34) & 41 & (66) & 67 & (34) \\
\hline & 2000-01 & 18 & (42) & 25 & (58) & 43 & (22) \\
\hline & Total & 69 & (35) & 126 & (65) & 195 & (100) \\
\hline
\end{tabular}

*Las residencias se agruparon en 3 áreas: médica, incluye residentes de medicina intema, pediatría y medicina ambulatoria; quirúrgica, incluye residentes de cirugía y obstetricia-ginecología; diagnóstica, incluye residentes de radiología, anatomía patológica y laboratorio clínico. Las residencias de subespecialidades se incluyeron dentro de las áreas médica 0 quirúrgica, según correspondiese. El rango de edad fue de 26 a 35 años en las residentes mujeres y de 23 a 39 en los hombres. **Se refiere a los residentes de las áreas primarias médica, pediátrica, quirúrgica y diagnóstica. 
Exposición a cursos y seminarios de cuidados paliativos, y autocalificación del nivel de formación alcanzado. En la Tabla 1 se observa que $45 \%$ de los residentes correspondían a médicos de especialidades médicas, $41 \%$ quirúrgicas y $14 \%$ a diagnósticas. El 76\% de los residentes, independientemente del área escogida, no tuvieron formación o cursos específicos en cuidados paliativos. Esta observación es importante si se considera que, aproximadamente $30 \%$ de los residentes encuestados, ya habían desarrollado una beca primaria de 3 años y se encontraban realizando durante este estudio una beca de subespecialidad. No hubo diferencias significativas entre los residentes provenientes de las universidades de Chile, Católica y las de provincias ni tampoco según sexo.
La Tabla 2 muestra el nivel subjetivo de preparación alcanzado en cuidados paliativos para la práctica profesional. La pregunta fue respondida sólo por los 75 residentes que estuvieron expuestos a docencia específica. Aproximadamente, la mitad de éstos consideró que su preparación era menor de $25 \%$ de lo considerado como necesario, $44 \%$, entre $25 \%$ y $50 \%$, y sólo $7 \%$ consideró que tenía una preparación en un nivel de 50 a $75 \%$ de lo necesario para desempeñarse apropiadamente en estas áreas. No hubo diferencias entre sexos, universidad de origen, ni entre grupos de especialidades.

Formación sobre aspectos antropológicos y éticos relacionados con la muerte. El $63 \%$ de los residen-

Tabla 2. Calificación del grado de preparación alcanzado en cuidados paliativos para la práctica profesional durante su formación médica (escala de $0 \%$ a $100 \%$ )

\begin{tabular}{|lcccc|}
\hline Grupo & \multicolumn{4}{c|}{ Grado de preparación alcanzado (\%) } \\
& $\leq 25$ & $\leq 50$ & $\leq 75$ & 100 \\
\hline Mujeres, n (\%) & $16(55)$ & $12(41)$ & $0(0)$ & $1(3)$ \\
Hombres, $\mathrm{n}(\%)$ & $19(41)$ & $18(39)$ & $8(17)$ & $1(2)$ \\
Total, n (\%) & $35(47)$ & $30(40)$ & $8(11)$ & $2(2)$ \\
\hline
\end{tabular}

Sólo 75 residentes contestaron esta pregunta, incluyendo los 57 que tuvieron formación en cuidados paliativos durante los estudios de pregrado.

Tabla 3. Calificación del nivel de preparación alcanzado en manejo de pacientes terminales y en aspectos psicológicos y antropológicos relacionados con la muerte (escala de $0 \%$ a $100 \%$ )

\begin{tabular}{|lcccc|}
\hline Actividad & $\leq 25 \%$ & \multicolumn{3}{c|}{ Nivel de preparación alcanzado } \\
& $\leq 50 \%$ & $\leq 75 \%$ & $\sim 100 \%$ \\
\hline $\begin{array}{l}\text { Manejo de pacientes terminales } \\
\text { Mujeres, n (\%) }\end{array}$ & $19(43)$ & $19(43)$ & $5(11)$ & $1(2)$ \\
Hombres, n (\%) & $32(40)$ & $27(34)$ & $16(20)$ & $5(6)$ \\
Total, n (\%) & $51(41)$ & $46(37)$ & $21(17)$ & $6(5)$ \\
& & & \\
Estudios de aspectos psicológicos y antropológicos sobre la muerte & $18(42)$ & $4(9)$ & $1(2)$ \\
Mujeres, n (\%) & $19(44)$ & $29(36)$ & $13(16)$ & $5(6)$ \\
Hombres, n (\%) & $32(40)$ & $47(40)$ & $17(14)$ & $6(5)$ \\
Total, n (\%) & $51(41)$ & & \\
\hline
\end{tabular}

La tabla resume las respuestas de los estudiantes que sí habían recibido formación en cuidados paliativos durante los estudios de pregrado. 
tes no había recibido formación específica en manejo de pacientes moribundos $\mathrm{y}$, aproximadamente $40 \%$, tampoco habían tenido clases de psicología y antropología de la muerte. Se destaca que cada residente de las especialidades médicas con más de un año de residencia, había sido tratante de entre 4 y 15 fallecidos por año. La mitad de los residentes consideró que su formación fue menor a $25 \%$ de lo necesario y aproximadamente la mitad, consideró ese mismo nivel de calificación en formación, sobre aspectos psicológicos y antropológicos relacionados con la muer- te. Sin embargo, la calificación del grado de preparación en dar órdenes de no resucitar y suspensión de terapias fútiles fue claramente mejor, como se puede observar en la Tabla 4. No hubo diferencias entre sexos, universidad de origen, ni entre grupos de especialidades médicas.

Grado de capacitación alcanzado en control de síntomas y manejo de fármacos. En la Tabla 5 se señala la distribución de los residentes según su calificación en la preparación alcanzada en control de síntomas de pacientes con enfermedades cróni-

Tabla 4. D istribución de los residentes según autocalificación del grado de preparación alcanzado en dar órdenes de no resucitar y suspensión de terapias fútiles en pacientes con enfermedades incurables en fase terminal

\begin{tabular}{|lcccccr|}
\hline Característica & \multicolumn{7}{c|}{ Calificación según escala de 1 a 7* } \\
& $\begin{array}{c}1-2 \\
\mathrm{n}(\%)\end{array}$ & $\mathrm{n}(\%)$ & $\mathrm{n}(\%)$ & $\mathrm{n}(\%)$ & $\mathrm{n}(\%)$ & Nota: $\mathrm{x} \pm$ DS \\
\hline Dar orden de no resucitar & $26(13)$ & $15(7)$ & $20(11)$ & $40(20)$ & $98(49)$ & $5,0 \pm 1,2$ \\
Suspender terapias fútiles & $20(11)$ & $15(7)$ & $26(13)$ & $47(23)$ & $85(43)$ & $5,0 \pm 1,5$ \\
\hline
\end{tabular}

*1-2= deficiente; $3=$ insuficiente; $4=$ regular; $5=$ más que regular; $6=$ bueno; $7=$ muy bueno. No se encontraron diferencias significativas entre sexos ni especialidades.

Tabla 5. D istribución de los residentes según autocalificación del grado de preparación alcanzado en control de síntomas en pacientes con enfermedades crónicas incurables en fase terminal

\begin{tabular}{|c|c|c|c|c|c|c|}
\hline \multirow[b]{2}{*}{ Síntomas } & \multicolumn{6}{|c|}{ Calificación según escala de 1 a 7* } \\
\hline & $\begin{array}{c}1-2 \\
\text { n (\%) }\end{array}$ & $\begin{array}{c}3 \\
\text { n (\%) }\end{array}$ & $\begin{array}{c}4 \\
\mathrm{n}(\%)\end{array}$ & $\begin{array}{c}5 \\
\mathrm{n}(\%)\end{array}$ & $\begin{array}{c}6-7 \\
n(\%)\end{array}$ & Nota $\mathrm{x} \pm \mathrm{DS}$ \\
\hline \multicolumn{7}{|l|}{ Sistémicos } \\
\hline Dolor & $5(3)$ & $10(5)$ & $20(10)$ & $83(41)$ & $83(41)$ & $5,2 \pm 1,0$ \\
\hline Astenia-caquexia & $68(32)$ & $37(18)$ & $47(23)$ & 38 (19) & $15(8)$ & $3,3 \pm 1,3 \#$ \\
\hline Anorexia & $64(32)$ & $37(18)$ & $47(23)$ & $38(19)$ & $15(8)$ & $3,4 \pm 1,3 \#$ \\
\hline \multicolumn{7}{|l|}{ Gastrointestinales } \\
\hline Náuseas & $12(6)$ & $17(9)$ & $21(10)$ & $74(37)$ & $77(38)$ & $4,9 \pm 1,2$ \\
\hline Constipación & $10(5)$ & $11(6)$ & $32(16)$ & $61(30)$ & $87(43)$ & $5,0 \pm 1,2$ \\
\hline Diarrea & $16(8)$ & $17(9)$ & $35(18)$ & $71(36)$ & $56(29)$ & $4,7 \pm 1,3$ \\
\hline \multicolumn{7}{|l|}{ Psiquiátricos } \\
\hline Angustia & $26(13)$ & $19(10)$ & $45(23)$ & $47(23)$ & $63(32)$ & $4,5 \pm 1,4$ \\
\hline Depresión & $26(13)$ & $20(11)$ & $48(26)$ & $46(25)$ & $47(25)$ & $4,5 \pm 1,4$ \\
\hline Delirio & $63(32)$ & $24(12)$ & $34(17)$ & $39(20)$ & $40(20)$ & $3,8 \pm 1,7 \#$ \\
\hline
\end{tabular}

$* 1-2=$ deficiente; $3=$ insuficiente; $4=$ regular; $5=$ más que regular; $6=$ bueno; $7=$ muy bueno.

$\# \mathrm{p}<0,05$ comparado con dolor. No se encontraron diferencias significativas entre sexos ni especialidades. 
cas incurables en fase terminal. El nivel de preparación parece relativamente adecuado en manejo del dolor, ya que $84 \%$ se puso una calificación igual o superior a 5. El $70 \%$ se consideró con una calificación de 40 inferior en el tratamiento de la astenia. En relación a síntomas digestivos, 75\% de los residentes se calificó con una nota igual 0 superior a 5 en manejo de la náusea, constipación y diarrea. En cuanto a los síntomas psiquiátricos, es interesante destacar que $56 \%$ de los residentes se calificó con 5 o $>5$ en el manejo de la angustia y el resto consideró que su calificación era sólo de 40 $<4$; algo similar ocurrió con el tratamiento de la depresión. Más de $60 \%$ consideró que su formación era insuficiente en el manejo del delirio. No se encontraron diferencias según sexo, universidad de origen, 0 especialidades.

El manejo de algunos de los fármacos frecuentes de usar, en el contexto de los cuidados paliativos, incluidos opioides, antináuseas, ansiolíticos y antidepresivos se autocalificó con una nota promedio de aproximadamente 5 .

Opinión sobre la importancia de los cuidados paliativos para su formación médica. Contrasta en general con los datos anteriores, el grado de importancia que atribuyeron los residentes a la medicina y los cuidados paliativos en su formación profesional, ya que $88 \%$ la consideró como importante o muy importante. En cuanto a cómo los residentes perciben que debieran ser la formación en control de síntomas y cuidados paliativos, $62 \%$ señaló que éstos debieran entregarse en forma de un módulo o curso específico multidisciplinario y 23\% consideró que los contenidos deberían ser entregados a lo largo del currículo clásico. Sólo 12\% sugirió que debiera ser durante el curso de oncología.

\section{DisCUSIÓN}

Este trabajo demuestra que la percepción de los residentes de una de las escuelas de postgrado más importante del país, respecto de su formación en medicina paliativa es insuficiente $y$, en algunas áreas, deficiente. Esto contrasta con los cambios curriculares implementados en la última década, tanto en Chile como en el extranjero. Estos cambios han sido, en general, orientados a cubrir las áreas llamadas humanistas de la formación y que se relacionan con las competencias necesarias para la atención médica general y, en especial, a pacientes portadores de enfermedades graves y avanzadas y de sus familias. Los objetivos y contenidos específicos han sido incluidos en cursos de ética clínica, antropología, geriatría, teología y de comunicación, procurando reforzar así las áreas psicológicas, sociales y espirituales propias de la formación médica $3,23,24,33$. Las áreas relacionadas con control de síntomas físicos tuvieron una buena evaluación, como también ocurrió con los aspectos éticos relacionados con la suspensión de terapias fútiles o dar órdenes de no resucitar, lo cual es coincidente con la introducción de cursos obligatorios de bioética y ética clínica en las últimas dos décadas en las escuelas de medicina ${ }^{3-10}$. No ocurrió lo mismo con las áreas psicológicas y antropológicas propias de la medicina paliativa relacionadas con el sufrimiento de la persona que enfrenta una enfermedad incurable 0 ve vecina su muerte ${ }^{1}$. Ello porque probablemente los cursos lectivos del área humanista impartidos durante los primeros años de carrera no sirven para cubrir estas áreas de la medicina clínica, las cuales pueden ser efectivamente incorporadas, sólo a través de ejercicios prácticos de relación de ayuda, comunicación, discusiones de pequeño grupo junto a profesores de clínica capacitados y tutorías personalizadas ${ }^{33}$.

Este estudio puede tener limitaciones. Quizás la más importante es el valor que pudiéramos arbitrariamente dar a las respuestas de los residentes para medir el grado de preparación alcanzado en medicina paliativa. Es posible que la percepción de las capacidades específicas de los residentes no tenga correlación significativa con la competencia real evaluada por terceros. Sin embargo, tres líneas de evidencia apoyan la hipótesis de que los resultados del estudio demuestran el nivel de preparación alcanzado en estas áreas. Primero, hay evidencias de que los residentes son tan capaces como sus docentes en la predicción de sus calificaciones ${ }^{34}$.

Segundo, los estudiantes tienden a subvalorar el grado de preparación y conocimientos comparado con sus docentes ${ }^{35}$. Tercero, las encuestas de autopercepción con evaluaciones altas se correlacionan positivamente con las calificaciones objetivas altas alcanzadas por los estudiantes ${ }^{36}$. 
En los últimos 20 años, la medicina paliativa ha evolucionado enormemente. Desde sus inicios, como un movimiento médico-social no alineado con la medicina académica, éstos han crecido hasta ser finalmente aceptada por los principales centros académicos mundiales ${ }^{24}$. La Organización Mundial de la Salud ${ }^{2}$, la Asociación Americana de Oncología Clínica (ASCO), la Academia Nacional de Ciencias de Estados Unidos, la Asociación Europea del Cáncer y un gran número de organizaciones médicas, científicas y gubernamentales han enfatizado la necesidad de promover la medicina y cuidados paliativos en la enseñanza médica $^{3,23-25,33}$. La relevancia de estos temas alcanza también las áreas de gestión económicoadministrativa, especialmente la preocupación creciente por los gastos innecesarios de los últimos meses de vida de una proporción muy importante de las personas que fallecen en los centros asistenciales estatales y privados. Esta situación tiene un fuerte impacto negativo sobre los presupuestos de salud de países desarrollados, como es el caso por ejemplo, de Estados Unidos de Norteamérica, considerando los enormes recursos que se gastan en las personas con enfermedades incurables durante su último año de vida ${ }^{37,38}$.

Los resultados de este estudio tienen especial relevancia para la medicina chilena y la atención de salud requerida por nuestro país, debido al envejecimiento progresivo de la población y la consiguiente incorporación de un universo mayor de personas expuestas a patologías crónicas incu-

\section{REFERENCIAS}

1. SAUNDERS DC. Principles of symptom control in terminal care. Med Clin North Am 1982; 66: 1169-83.

2. Cancer Pain Relief and Palliative Care. Technical report series 804. Geneva: World Health Organization, 1990.

3. Doyle D, Hanks GW, MacDonald N. Introduction. En: Doyle D, Hanks GW, MacDonald N. Oxford Textbook of Palliative Medicine. Oxford UK: Oxford University Press 1999; 3-11.

4. LoNGO DL. Palliative care in management of terminally ill patients. En: Fauci AS, Braunwald E, Isselbacher KJ, Wilson JD, Martin JB, Kasper DL, Hauser SL, Longo DL, Eds. Harrison's 14th Edition CD-ROM update: Approach to the patient with cancer, McGraw-Hill, New York. 2000; capítulo 81. rables. Las amenazas actuales y futuras respecto de la calidad de atención médica de los enfermos incurables, también se encuentran en las propias escuelas de medicina y en las estructuras hospitalarias universitarias. En éstas, el control de síntomas y el manejo multidisciplinario del paciente incurable que enfrenta una enfermedad catastrófica, por ejemplo un cáncer epitelial diseminado, no existe generalmente estructurado de manera adecuada para cumplir asistencialmente bien y para cumplir las funciones docentes.

Los datos de este trabajo deben llamar la atención de las autoridades de salud y las universitarias, las cuales deberán promover la formación de recursos docentes apropiados, estructuras asistenciales específicas y suficientes, para una atención personalizada, humana e integral. Será necesario modificar apropiadamente las áreas curriculares, especialmente los módulos docentes relacionados con cáncer, medicina interna y familiar, cirugía, geriatría, pediatría y en general, todas las subespecialidades, además de una específica y particular preocupación por la familia durante la enfermedad y el período del duelo. No menos relevante y urgente es el desarrollo de protocolos clínicos relacionados con el control apropiado de síntomas del paciente incurable, basados en evidencias científicas sólidas, y además, desarrollar estudios de análisis de costo-efectividad para fundamentar el uso racional de analgesia eficaz y de fácil administración como la morfina oral, no siempre disponibles en el sistema público.

5. CASSEL EJ. The Nature of Suffering and the Goals of Medicine. N Engl J Med 1988; 306: 639-45.

6. CASSEL EJ. The Nature of Suffering and the Goals of Medicine. New York, NY: Oxford University Press; 1994.

7. Bretscher ME, CREAgan ET. Understanding suffering: what palliative medicine teaches us. Mayo Clin Proc 1997; 72: 785-91.

8. Callahan D. Death and the research imperative. $\mathrm{N}$ Engl J Med 2000; 342: 654-6.

9. Weiss SC, Emanuel LL, Fairclough DL, Emanuel EJ. Understanding the experience of pain in terminally ill patients. Lancet 2001; 357: 1311-5.

10. Meyers FJ, Linder J. Simultaneous Care: Disease treatment and palliative care throughout illness. J Clin Oncol 2003; 21: 1412-5. 
11. Eluershaw J, Ward C. Care of the dying patient: the last hours or days of life. BMJ 2003; 326: 30-4.

12. Reyes BH, KaufFmann QR, AndResen HM. Las Escuelas de Medicina Chilenas, en los albores del siglo XXI. Rev Méd Chile 1997; 125: 781-2.

13. RosSELOT EJ. Reorganización en la educación médica. Aspectos esenciales de la reestructuración de la Facultad de Medicina de la Universidad de Chile. Rev Méd Chile 1997; 125: 783-91.

14. SchiappaCASSE EF. Propósitos y objetivos de la formación de médicos en la Facultad de Medicina de la Universidad de Concepción. Rev Méd Chile 1997; 125: 792-5.

15. Rosso RP, Velasco FN, Moreno BR. Reforma del curriculum de pregrado en la Escuela de Medicina de la Pontificia Universidad Católica de Chile: Objetivos, metodología y estado de avance. Rev Méd Chile 1997; 125: 796-807.

16. SABaH JD. Propósitos y objetivos de la formación de médicos en la Universidad de Valparaíso. Rev Méd Chile 1997; 125: 808-13.

17. Groв BC. La Escuela de Medicina de la Universidad Austral de Chile. Un compromiso regional. Rev Méd Chile 1997; 125: 814-7.

18. Stockins FB, Pantoja MM. Facultad de Medicina de la Universidad de La Frontera. Rev Méd Chile 1997; 125: 818-22.

19. ORREGo VF. Educación médica en la Universidad de los Andes. Rev Méd Chile 1997; 125: 823-6.

20. Mardones RF. La Facultad de Ciencias Médicas de la Universidad de Santiago de Chile. Rev Méd Chile 1997; 125: 827-33.

21. Merman AC, Gunn DB, Dickinson GE. Learning to care for the dying: A survey of medical schools and a model course. Acad Med 1991; 66: 35-8.

22. Biwngs JA, Bцоск S. Palliative care in undergraduate medical education. Status report and future directions. JAMA 1997; 278: 733-8.

23. Scott JF, MacDonald N, Mount BM. Palliative medicine education. En: Oxford Textbook of Palliative Medicine. 2nd ed., New York, Oxford University Press, 1999; 1169-99.

24. Stjernswärd J, PampaLona S. Palliative Medicine. A global perspective. En: Oxford Textbook of Palliative Medicine. 2nd ed., New York, Oxford University Press 1999; 1227-45.

25. Oneschuk D, Hanson J, Bruera E. An international survey of undergraduate medical education in palliative medicine. J Pain Symptom Manage 2000; 20: 174-9.

26. Laison Committee on Medical Education (LCME). Accrediting authority for medical education pro- grams leading to the MD degree in US and Canadian medical schools. http://www.lcme.org. Acceso Mayo, 2003.

27. ARNoLD R. The challenges of integrating palliative care into postgraduate training. Innovations in end of life Care 2002: 4: www.edc.Org/lastacts. Acceso mayo, 2003.

28. Von Gunten CF. Secondary and tertiary palliative care in US hospitals. JAMA 2002; 287: 875-81.

29. Rico MA. Palliative care in Chile. Eur J Pall Care 1997; 4: 138-9.

30. Nervi F, Guerrero M, Reyes MM, Nervi B, Cura A, Chávez M et aL. Symptom control and Palliative Care in Chile. J Pain Pal Care Pharmacotherapy, 2003 en prensa.

31. A Wordd Bank Country Study. Chile. The adult health policy challenge. The World Bank, Washington DC, 1995.

32. Ministerio de Salud. Departamento de Epidemiología. Causas de Mortalidad, 1998. http://minsal.cl. Acceso Mayo, 2003.

33. Jones R, Higgs R, De Angelis C, Prideaux D. Changing face of medical curricula. Lancet 2001; 357: 699-703.

34. Hawkins RE, Sumption KF, Gaglone MM, Holmboe ES. The in training examination in internal medicine: resident perceptions and lack of correlation between resident scores and faculty predictions of resident performance. Am J Med 1999; 106: 206-10.

35. FincER RM, Lewis LA, KuSKe TT. Relationships of interns' performances to their self assessments of their preparedness for internship and to their academic performances in medical school. Acad Med 1993; 68: S47-S50.

36. Shubert A, Tetzlaff JE, Tan M, Ryckman JV, Mascha E. Consistency, inter rater reliability and validity of 441 consecutive mock oral examinations in anesthesiology: implications for use as a tool for assessment of residents. Anesthesiology 1999; 91: 288-98.

37. CasSel CK, VladeCK BC. ICD-9 Code for palliative or terminal care. N Engl J Med 1996; 335: 1232-4.

38. MATHERLEE K. Managing advanced illness: a quality and cost challenge to Medicare, Medicaid, and private insurers. National Health Policy Forum Issue Brief. 2002; № 779. http://www.nhpf.org/ about/about.htm. Acceso, Junio, 2003.

\section{Agradecimientos}

Los autores agradecen los comentarios y sugerencias de los Drs. Sergio Jacobelli y Vicente Valdivieso. 